Revista Brasil. Bot., V.31, n.3, p.389-398, jul.-set. 2008

\title{
Biologia reprodutiva de Rourea induta Planch. (Connaraceae), uma espécie heterostílica de cerrado do Brasil Central
}

\author{
EDDIE LENZA ${ }^{1,2,5}$, JOICE NUNES FERREIRA ${ }^{1,3}$, HÉLDER CONSOLARO $^{1} \mathrm{e}$ \\ FABIANA DE GOIS AQUINO ${ }^{1,4}$
}

(recebido: 24 de maio de 2007; aceito: 29 de maio de 2008)

\begin{abstract}
Reproductive biology of Rourea induta Planch. (Connaraceae), a heterostylous species from the cerrado of Central Brazil). Heterostyly is a floral polymorphism usually associated to intramorph-incompatible mating system. We evaluated reproductive aspects of heterostyly in a Rourea induta Planch. population in a cerrado area of Brasília, Central Brazil. Analyses of the floral biology, breeding systems, fruit set and maturation, seed germination, reproductive phenology and floral visitors of were conducted. The flowers are small (about $11 \mathrm{~mm}$ diameter), with single morphology, and have five pistils and two sets of five stamens of unequal lengths. The average lengths of stamens and pistils were significantly different between thrum and pin morphs. However, complete reciprocal herkogamy was not found between stamen and pistil lengths of the two floral morphs. Both morphs are intramorph-incompatible. However, thrums are completely self-incompatible while pins are partially self-compatible. In spite of the differences in the floral morphology and breeding system, the two morphs had similar reproductive success in natural condition. The two floral morphs did not differ significantly in terms of fruit setting and maturation or seed germination rate. Rourea induta produced abundant flowers daily over two short events ("pulsed bang" flowering) and most plants in the population flowered synchronously. Many species of insects, mainly small social bees, were observed visiting the flowers. Our results suggest that partial self-incompatibility, intramorph-incompatibility, and differences between morphs in lengths of stamens and pistils reduced autogamy levels in $R$. induta. Ultimately, the higher fruit setting by intermorph pollination promotes isoplety and indicates that sexual reproduction in $R$. induta population depends on pollinators services.
\end{abstract}

Key words - breeding systems, distyly, melittophily, reproductive success, savanna

RESUMO - (Biologia reprodutiva de Rourea induta Planch. (Connaraceae), uma espécie heterostílica de cerrado do Brasil Central). A heterostilia é um polimorfismo floral geralmente associado a sistemas genéticos de intramorfo incompatibilidade. Nós avaliamos aspectos reprodutivos da heterostilia de uma população de Rourea induta Planch. em uma área de cerrado de Brasília, DF. Foram conduzidos estudos sobre sua biologia floral, sistema reprodutivo, produção e maturação de frutos, germinação de sementes, fenologia reprodutiva e visitantes florais. As flores são pequenas (11 mm de diâmetro), com morfologia simples e possuem dois grupos de cinco estames de comprimentos diferentes e cinco pistilos. O comprimento médio de estames e pistilos diferiu significativamente entre o morfo brevistilo e o longistilo. No entanto, não houve hercogamia recíproca completa entre os dois morfos florais. Os dois morfos são intramorfo incompatíveis, mas o morfo brevistilo é completamente auto-incompatível enquanto o longistilo é parcialmente autocompatível. Apesar das diferenças na morfologia floral e no sistema reprodutivo entre os morfos, estes apresentam igual sucesso reprodutivo em condições naturais, uma vez que a produção e a maturação de frutos por planta e a taxa de germinação das sementes não diferiram significativamente. A população de $R$. induta apresentou floração tipo "pulsed bang" com alta sincronia intra-individual e inter-individual de floração. Grande variedade de insetos, principalmente pequenas abelhas sociais, foi observada visitando as flores. Nossos resultados sugerem que a auto-incompatibilidade parcial, a incompatibilidade intramorfo e as diferenças nas alturas dos verticilos reprodutivos entre os dois morfos reduzem os níveis de autogamia em $R$. induta. Em última análise, a maior produção de frutos por polinização intermorfo, promoveu a razão isoplética na população estudada e indicou que $R$. induta é dependente dos polinizadores para a reprodução sexuada.

Palavras-chave - distilia, melitofilia, savana, sistemas reprodutivos, sucesso reprodutivo

\section{Introdução}

A heterostilia é um polimorfismo floral geneticamente controlado, sendo caracterizada pela hercogamia recíproca

1. Universidade de Brasília, Instituto de Ciências Biológicas, Departamento de Ecologia, Programa de Pós-Graduação em Ecologia, Caixa Postal 04457, 70919-970 Brasília, DF, Brasil.

2. Endereço atual: Universidade do Estado de Mato Grosso, Campus Nova Xavantina, Departamento de Ciências Biológicas, BR158, km 148, Caixa Postal 08, 78690-000 Nova Xavantina, MT, Brasil. entre morfos florais e por um sistema genético de incompatibilidade intramorfo (Vuilleumier 1967, Ganders 1979a, Barrett et al. 2000, Barrett 2002). O polimorfismo floral pode manifestar-se na condição distílica (morfos

3. Endereço atual: Empresa Brasileira de Pesquisa Agropecuária, Embrapa Amazônia Oriental, Caixa Postal 48, 66017-970 Belém, PA, Brasil.

4. Endereço atual: Empresa Brasileira de Pesquisa Agropecuária, Embrapa Cerrados, Caixa Postal 08223, 73310-970 Planaltina, DF, Brasil.

5._Autor para correspondência: eddielenza@ yahoo.com.br 
brevistilos e longistilos) ou tristílica (morfos brevistilos, medistilos e longistilos) (Ganders 1979a).

Em espécies distílicas, o polimorfismo floral aumenta a precisão na transferência de pólen (Darwin 1877, Lloyd \& Webb 1992, Barrett 2002) e promove a polinização cruzada entre os dois morfos florais (Vuilleumier 1967, Ganders 1974, Ganders 1979a, Ganders et al. 1985). O sistema genético de incompatibilidade manifesta-se de forma que apenas cruzamentos intermorfos resultam na formação de frutos (Lewis 1943, Barrett 1992). Essas características morfológicas e genéticas caracterizam espécies tipicamente distílicas e são fortemente associadas por meio de uma ligação entre os loci gênicos que as controlam, formando o que é chamado de supergene (Ganders 1979a). Além do mais, os cruzamentos intermorfos são responsáveis pela razão equilibrada entre os dois morfos dentro de populações de plantas distílicas, ou isopletia (Ganders 1979a, Sobrevila et al. 1983, Shore \& Barrett 1985, Lloyd \& Webb 1992, Barrett et al. 2000).

No entanto, podem ocorrer variações nos sistemas de cruzamentos de espécies distílicas, devido à evolução de diferentes graus de autocompatibilidade entre espécies e morfos (Baker 1966, Ganders 1979a, Sobrevila et al. 1983, Barrett \& Cruzan 1994) e à perda total ou parcial da hercogamia recíproca (Ganders 1979a, Consolaro et al. 2005). Estas mudanças genéticas e morfológicas podem aumentar as taxas de polinização intramorfo, conduzindo à anisopletia (razão não equilibrada entre os dois morfos na população) (Lloyd \& Webb 1992, Barrett et al. 2000).

Vinte e oito famílias de Angiospermas possuem espécies heterostílicas, entre elas a família Connaraceae (Barrett et al. 2000, Lloyd \& Webb 1992). Todavia, esta família se diferencia das demais em função de seus representantes possuírem diferentes graus na expressão da heterostilia (Ganders 1979a, Lemmens 1989). Segundo Lemmens (1989) a tristilia em Connaraceae é uma característica primitiva e ocorrem oito variações heterostílicas na família, que podem ser derivadas da tristilia. A distilia com 10 estames férteis é o tipo mais comum na família e surgiu a partir da perda do morfo intermediário de uma espécie tristílica ancestral (Lemmens 1989). O gênero Rourea é considerado tipicamente distílico (Ganders 1979a, Lemmens 1989), sendo caracterizado por flores com cinco pistilos apocárpicos e dois grupos de estames com tamanhos diferentes (Lemmens 1989).

No Cerrado, a família Connaraceae é representada por sete espécies arbustivo-arbóreas distribuídas nos gêneros Rourea e Connarus (Mendonça et al. 1998). A despeito da ocorrência de distilia em Connaraceae, não há estudos específicos ou experimentais sobre sistemas reprodutivos das espécies desta família no Cerrado.
Este trabalho teve como objetivo analisar aspectos reprodutivos de uma população da espécie distílica Rourea induta Planch. em uma área de cerrado do Brasil Central. Foram analisados a morfologia floral, o sistema reprodutivo, a produção e maturação de frutos, a germinação de sementes e observados os eventos fenológicos reprodutivos e os visitantes florais a fim determinar os mecanismos responsáveis por garantir a reprodução sexuada da espécie.

\section{Material e métodos}

Área de estudo e espécie estudada-O estudo foi desenvolvido em uma área de cerrado sentido restrito na Reserva Ecológica do IBGE (Recor), Distrito Federal, Brasil (1555'06"-

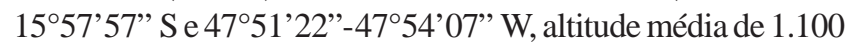
metros). Na Recor, a temperatura anual varia entre $18^{\circ} \mathrm{C}$ e $22{ }^{\circ} \mathrm{C}$ e a pluviosidade média anual é cerca de $1.400 \mathrm{~mm}$, com uma estação chuvosa de outubro a abril e uma estação seca entre maio e setembro (Estação Climatológica da Recor).

Rourea induta é uma espécie arbustiva com até 4 metros de altura comum no cerrado, sendo típica em fisionomias de campo sujo e cerrado sentido estrito no Distrito Federal (Forero 1983, Milhomens \& Proença 2002, Nunes et al. 2002). Suas flores pálido-amareladas estão dispostas em panículas e apresentam dois grupos de estames com diferentes tamanhos (Milhomens \& Proença 2002).

O trabalho de campo foi realizado nos eventos reprodutivos de 1998, 2000 e 2005. No primeiro evento, foi analisada a morfologia e a biologia floral e observados os visitantes florais. No segundo, foi caracterizada a fenologia reprodutiva. No terceiro, foi novamente analisada a fenologia reprodutiva, bem como avaliados os sistemas reprodutivos e realizados testes de germinação de sementes.

Morfologia floral, sistemas reprodutivos e germinação de sementes - Foram realizadas buscas e marcação de indivíduos dos dois morfos florais (brevistilo e longistilo) na área de estudo. Foram determinados por meio de observações diretas no campo, o número e a posição dos verticilos florais de cada morfo (60 flores por morfo distribuídas em seis indivíduos). Foram coletadas e fixadas flores em FAA em álcool $50 \%$ e, posteriormente, tomadas medidas de suas estruturas florais em laboratório com auxílio de paquímetro com precisão de $0,5 \mathrm{~mm}$. Para cada morfo, foram medidos dois pistilos e quatro estames (dois maiores e dois menores) em quatro flores de quatro indivíduos, totalizando 32 verticilos de cada tipo por morfo. Foi calculado o Índice de Reciprocidade (IR) para os dois morfos florais, de acordo com Richards \& Koptur (1993): IR = (altura da antera - altura do estigma recíproco)/(altura da antera + altura do estigma recíproco). Segundo esses autores, ocorre hercogamia recíproca quando o IR dos dois morfos é próximo de zero.

Testes de sistema reprodutivo foram realizados em 35 indivíduos, sendo 18 longistilos e 17 brevistilos, seguindo 
o mesmo procedimento adotado em estudos sobre sistema reprodutivo de espécies distílicas (Bawa \& Beach 1983, Richards \& Koptur 1993, Barros 1998). Foi calculado o Índice de Auto-incompatibilidade (IAI), determinado pela razão entre a produção de frutos por autopolinização e por polinização cruzada intermorfo (Bullock 1985), bem como o Índice de Eficiência Reprodutiva dos polinizadores (IER), dado pela razão entre a produção de frutos do tratamento controle e da polinização cruzada intermorfo (Ruiz \& Arroyo 1978).

Os testes de germinação foram conduzidos com sementes não danificadas e sem sinais de predação, coletadas de 11 indivíduos brevistilos e 13 indivíduos longistilos $(n=310 \mathrm{e}$ $n=210$ sementes, respectivamente) e seguindo o protocolo sugerido por Oliveira et al. (1989). A avaliação da germinação foi realizada em censos semanais durante quatro semanas, sendo consideradas como germinadas as sementes que emitiram radícula.

Biologia floral, fenologia e visitantes florais - A presença de néctar e de pólen foi verificada em flores utilizadas nos estudos de morfologia floral e por observações diretas das flores no campo. O início da antese foi determinado pela marcação de 240 botões (40 botões de duas inflorescências e de seis indivíduos). Durante cinco dias, as inflorescências foram observadas a cada duas horas, no intervalo entre $8 \mathrm{~h} 00$ e 18h00. A longevidade das flores foi determinada para 211 botões florais, marcados em pré-antese e distribuídos em seis indivíduos. Os botões florais foram acompanhados em intervalos de 24 horas desde a antese até a senescência floral, caracterizada pelo murchamento e escurecimento da flor.

No estudo fenológico no ano de 2000 foi determinada a presença e ausência das fenofases de floração, produção e maturação dos frutos em 37 indivíduos (18 brevistilos e 19 longistilos). Em 2005 foi estimada a intensidade de floração em 69 indivíduos (32 brevistilos e 37 longistilos), de acordo com Fournier (1974). Os indivíduos foram marcados e morfotipados ao longo de um transecto de 800 metros e foi determinada a razão isoplética entre os dois morfos. Com base nas estimativas individuais, foi calculada a Intensidade de Floração da População (IFP) (ver Fournier 1974, Bencke \& Morellato 2002) para os dois morfos. O pico de floração foi definido pela porcentagem de indivíduos em flor ou pela IFP, igual ou superior a $50 \%$. O padrão de floração foi caracterizado de acordo com Proença \& Gibbs (1994).

No evento reprodutivo de 2000 , foi contado o número de frutos maduros produzidos pelos 37 indivíduos ao longo de todo período de maturação. Uma vez contados, os frutos eram marcados com cola colorida para evitar recontagem nos censos seguintes. No evento de 2005, as observações fenológicas foram encerradas no início da maturação dos frutos. Os frutos imaturos e maduros produzidos pelos 69 indivíduos acompanhados foram contados e considerados conjuntamente como "frutos potencialmente maduros". No evento de 2005, alguns indivíduos produziram grande quantidade de frutos, o que impossibilitou o uso da mesma metodologia adotada no evento de 2000.
Os visitantes florais com hábito diurno foram coletados em uma planta de cada morfo durante o pico de floração da população que se estendeu por dois dias. As coletas foram realizadas em dois intervalos pela manhã (entre 8h00 e 9h00; entre $10 \mathrm{~h} 30$ e 11h30) e em dois intervalos à tarde (entre 14h30 e 15h30; entre $17 \mathrm{~h} 00$ e 18h00). Anteriormente à coleta, foi observado ainda o comportamento de visita das espécies. Foram tomadas medidas de comprimento do corpo e as espécies classificadas como pequenas ou grandes $(\leq 12 \mathrm{~mm}$ e $>12 \mathrm{~mm}$, respectivamente) de acordo com Oliveira \& Gibbs (2000). Os visitantes coletados foram sacrificados com câmara mortífera, montados com alfinetes entomológicos, etiquetados e identificados por especialistas.

Análises estatísticas - A comparação do comprimento dos verticilos reprodutivos entre as duas formas florais foi feita por Análise de Variância (ANOVA) dos dados ranqueados para dois fatores, forma floral (brevistila e longistila) e tipo de verticilo (estame grande, estame pequeno e pistilo). Foi aplicado o teste de Mann-Whitney comparando os dois morfos quanto ao número de frutos maduros por planta no ano de 2000 e quanto ao número de frutos potencialmente maduros produzidos por indivíduo ao final do período reprodutivo de 2005. Teste exato de Fisher para aproximação de $\chi^{2}$ foi aplicado para testar a razão isoplética dos morfos florais na população; comparar a freqüência de sementes germinadas entre os dois morfos; comparar freqüência de formação de frutos entre autopolinização manual e polinização cruzada intermorfo e entre controle e polinização cruzada intermorfo; e comparar as freqüências de coletas dos visitantes florais entre o período matutino e o vespertino.

\section{Resultados}

Morfologia floral, sistema reprodutivo e germinação de sementes - As flores de $R$. induta são pentâmeras, actinomorfas, gamossépalas, possuem corola dialipétala branca e medem cerca de $11 \mathrm{~mm}$ de diâmetro. $\mathrm{O}$ androceu é composto por 10 estames monadelfos e soldados na porção basal, tendo um grupo com cinco estames maiores e outro com cinco menores. O gineceu é composto por cinco pistilos apocárpicos com dois óvulos por carpelo. Os frutos são do tipo folículo, com ápice levemente apiculado, de cor verde quando imaturos e alaranjados a vermelhos quando maduros. Produzem uma semente de cor negra com arilo amarelado na sua porção inferior.

A população estudada apresenta dois morfos florais (figura 1). Não houve diferença significativa entre a frequiência de indivíduos brevistilos e longistilos (18 e 19 no ano de 2000: $\chi_{0,05,1}^{2}=0, P=1 ; 32$ e 37 no ano de 2005: $\left.\chi_{0,05,1}^{2}=0,232, P=0,6301\right)$. As flores brevistilas apresentam os pistilos localizados abaixo do menor grupo estaminal (figura 1A), enquanto os pistilos das flores longistilas estão posicionados acima do maior grupo 

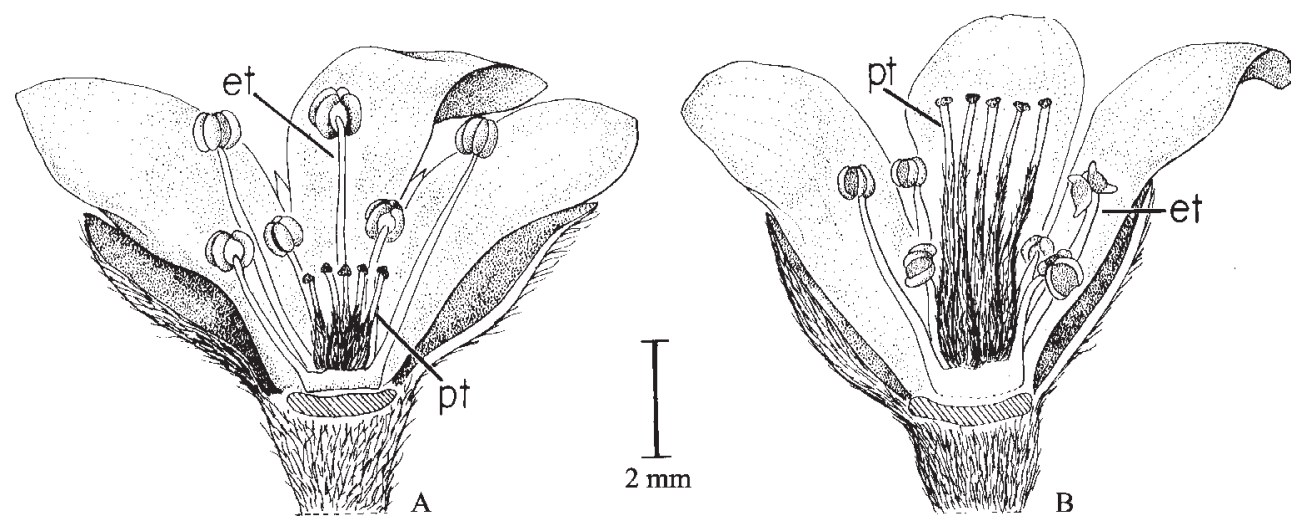

Figura 1. Flores de Rourea induta em uma área de cerrado sentido restrito na Reserva Ecológica do IBGE, Distrito Federal, Brasil. Duas sépalas e duas pétalas foram removidas. $(\mathrm{A}=$ flor brevistila; $\mathrm{B}=$ flor longistila; $\mathrm{pt}=$ pistilo; et $=$ estame).

Figure 1. Rourea induta flowers in a cerrado strictu sensu area at Reserva Ecológica do IBGE, Distrito Federal, Brazil. Two sepals and two petals were removed. ( $\mathrm{A}=$ thrum flower; $\mathrm{B}=$ pin flower; $\mathrm{pt}=$ pistil; et $=$ stamens $)$.

estaminal (figura 1B). As flores brevistilas apresentam os dois grupos estaminais significativamente maiores que os respectivos grupos das flores longistilas, sendo a situação inversa encontrada para os pistilos $\left(F_{2,0,05}=\right.$ $272,6333, P<0,0001$ ) (tabela 1$)$. A hercogamia recíproca precisa ocorreu apenas entre os pistilos das flores longistilas e os estames do maior grupo estaminal das flores brevistilas $\left(F_{2,0,05}=272,6333, P=0,4229\right)$. Os índices de reciprocidade (IR) foram relativamente elevados, considerando-se tanto os estames grandes (brevistilo $=0,02$; longistilo $=0,33$ ) quanto os estames pequenos $($ brevistilo $=-0,13 ;$ longistilo $=0,15)$,

Tabela 1. Comparações entre os comprimentos $(\mathrm{mm})$ dos verticilos reprodutivos dos morfos brevistilos e longistilos de flores de Rourea induta (ANOVA dos dados ranqueados) em uma área de cerrado sentido restrito na Reserva Ecológica do IBGE, Distrito Federal, Brasil.

Table 1. Comparisons between lengths ( $\mathrm{mm}$ ) of reproductive verticils of thrum and pin morphs of a Rourea induta flowers (rank data ANOVA) in a cerrado strictu sensu area at Reserva Ecológica do IBGE, Distrito Federal, Brazil.

\begin{tabular}{lccc}
\hline $\begin{array}{l}\text { Tipo de } \\
\text { verticilo }(n)\end{array}$ & $\begin{array}{c}\text { Morfo } \\
\text { brevistilo } \\
\text { Média }( \pm s)\end{array}$ & $\begin{array}{c}\text { Morfo } \\
\text { longistilo } \\
\text { Média }( \pm s)\end{array}$ & $\begin{array}{c}\text { Teste de } \\
\text { Tukey } \\
P\end{array}$ \\
\hline $\begin{array}{l}\text { Estame } \\
\text { grande (32) }\end{array}$ & $4,85( \pm 0,44)$ & $4,19( \pm 0,58)$ & $<0,0001$ \\
pequeno (32) & $3,61( \pm 0,42)$ & $2,83( \pm 0,48)$ & $<0,0001$ \\
Pistilo (32) & $2,09( \pm 0,20)$ & $4,66( \pm 0,41)$ & $<0,0001$ \\
\hline
\end{tabular}

confirmando a ausência de reciprocidade completa entre os morfos florais.

Para o morfo brevistilo, a produção de frutos por polinização cruzada intermorfo foi significativamente maior do que aquela observada por autopolinização manual e por polinização cruzada intramorfo $\left(\chi_{1,0,05}^{2}=12,246\right.$, $P=0,0005$ e $\chi_{1,0,05}^{2}=9,7, P=0,0018$, respectivamente). Igualmente para o morfo longistilo, a produção de frutos foi significativamente maior na polinização cruzada intermorfo em relação à autopolinização manual e à polinização cruzada intramorfo $\left(\chi_{1,0.05}^{2}=8,777, P=0,0031\right.$ e $\chi_{1,0,05}^{2}=23,696, P<0,0001$, respectivamente), indicando que os dois morfos são auto-incompatíveis e intramorfo incompatíveis. No entanto, considerando-se o índice de auto-incompatibilidade, o morfo longistilo teve IAI igual a 0,42 , ou seja, dentro dos limites de autocompatibilidade parcial enquanto que o IAI do morfo brevistilo foi 0,07 , ou seja, correspondente a auto-incompatibilidade completa (tabela 2).

O tratamento controle no morfo brevistilo produziu significativamente mais frutos que o longistilo $\left(\chi_{1,0,05}^{2}=\right.$ 20,797, $P<0,0001)$. Além disso, o morfo brevistilo produziu significativamente mais frutos no controle do que em polinizações cruzadas intermorfo (IER = 1,53; $\chi_{1,0,05}^{2}=4,063, P=0,0438$ ), enquanto o morfo longistilo apresentou significativamente mais frutos originados nas polinizações cruzadas intermorfo do que no tratamento controle (IER $=0,57 ; \chi_{1,0,05}^{2}=7,341, P=0,0067$ ) (tabela 2 ).

A formação de frutos por apomixia foi nula no morfo brevistilo e baixa no morfo longistilo (5\%) (tabela 2), além disso, os frutos produzidos neste tratamento apresentaram desenvolvimento retardado em relação aos frutos formados 
Tabela 2. Produção de frutos oriundos de experimentos de polinização controlada em uma população de Rourea induta em uma área de cerrado sentido restrito na Reserva Ecológica do IBGE, Distrito Federal, Brasil.

Table 2. Fruit setting by controlled pollination experiments of a Rourea induta population in a cerrado strictu sensu area at Reserva Ecológica do IBGE, Distrito Federal, Brazil.

\begin{tabular}{lrrrrrrrr}
\hline \multirow{2}{*}{ Tratamento } & \multicolumn{3}{c}{ Morfo brevistilo } & & \multicolumn{3}{c}{ Morfo longistilo } \\
\cline { 2 - 3 } & Flores & Frutos & $\%$ & & Flores & Frutos & $\%$ \\
\hline Controle & 451 & 183 & 40,6 & & 369 & 93 & 25,2 \\
Polinização cruzada intermorfo & 64 & 17 & 26,6 & & 52 & 23 & 44,2 \\
Polinização cruzada intramorfo & 54 & 2 & 3,7 & & 58 & 2 & 3,4 \\
Autopolinização manual & 55 & 1 & 1,8 & & 80 & 15 & 18,8 \\
Apomixia & 127 & 0 & 0,0 & & 100 & 5 & 5,0 \\
Índice de Auto-incompatibilidade & & 0,07 & & & & & 0,42 & \\
Índice de Eficiência Reprodutiva & & 1,53 & & & & 0,57 \\
\hline
\end{tabular}

nos demais tratamentos e não amadureceram. Das 310 sementes de indivíduos brevistilos, 49,7\% germinaram, enquanto das 200 sementes de indivíduos longistilos, $42,5 \%$ germinaram. A frequiência de germinação não diferiu entre os dois morfos $\left(\chi_{0,05,1}^{2}=2,235, P=0,1349\right)$.

Biologia floral, fenologia e visitantes florais - A antese das 240 flores acompanhadas ocorreu em dois períodos, entre $08 \mathrm{~h} 00$ e $10 \mathrm{~h} 00$ (56\%) e entre $18 \mathrm{~h} 00$ e $08 \mathrm{~h} 00$ (34\%). Dez por cento dos botões observados foram abortados. A longevidade máxima das flores foi de 48 horas, sendo caracterizada pelo murchamento completo das pétalas, todavia em 24 horas após a antese os estames já apresentavam mudança na coloração.

A população estudada apresentou padrão de floração do tipo "pulsed bang" (Proença \& Gibbs 1994) com dois picos de aproximadamente uma semana ao longo dos meses de setembro a novembro. No primeiro pico todos os indivíduos floresceram intensamente, refletindo-se em altos valores de IFP. Nem todos os indivíduos floresceram durante o segundo pico e muitos destes indivíduos produziram pequeno número de flores e inflorescências, o que refletiu em valores mais baixos de IFP (figura 2).

Dos 37 indivíduos reprodutivos amostrados em 2000, 31 indivíduos (83,8\%; 15 brevistilos e 16 longistilos) iniciaram a produção de frutos entre setembro e outubro de 2000 e foi observada a presença de frutos maduros em 20 indivíduos (54,1\%; nove brevistilos e 11 longistilos) entre dezembro de 2000 e fevereiro de 2001. Já em 2005, os 69 indivíduos reprodutivos acompanhados iniciaram a formação de frutos e 43 indivíduos $(62,3 \%$; 22 brevistilos e 21 longistilos) deram início à maturação dos frutos em janeiro de 2006 (figura 2).
O número de frutos maduros produzidos por planta no ano de 2000 não diferiu significativamente entre os morfos brevistilo ( $n=32 ; 1,8$ frutos/planta) e longistilo $\left(n=91 ; 4,8\right.$ frutos/planta) $\left(\chi_{0,05,1}^{2}=0,013, P=0,908\right)$. No ano de 2005, o número de frutos "potencialmente maduros" produzidos não diferiu entre os morfos brevistilo $(n=4.671 ; 146$ fruto/planta) e longistilo $(n=$ 2.277; 61,5 frutos/planta) $\left(\chi_{0,05,1}^{2}=0,037, P=0,847\right)$.

As flores foram visitadas apenas por insetos, caracterizando entomofilia para esta espécie. Foram coletados 103 indivíduos, pertencentes a 21 espécies, com predominância de abelhas (86,4\% dos indivíduos e $47,6 \%$ das espécies). A atividade dos visitantes florais no período matutino, com 82 coletas, foi maior do que aquela observada no período vespertino, com 21 coletas $\left(\chi_{0,05,1}^{2}=34.954, P<0,0001\right)$. Quanto ao comprimento do corpo, 15 espécies $(71,4 \%)$ eram pequenas e seis $(28,6 \%)$ grandes. No grupo das abelhas, $90 \%$ das espécies eram pequenas. As espécies mais freqüentes foram Scaptotrigona postica, Paratrigona lineata, Apis mellifera, Trigona spinipes e Tetragona cf. clavipes, que representaram $81,6 \%$ do número total de indivíduos coletados (tabela 3 ).

Observações de campo realizadas durante a coleta dos visitantes permitem algumas generalizações a respeito do comportamento das abelhas, que consistiu em pousar sobre uma flor e caminhar sobre outras flores da mesma inflorescência. Com vôos curtos, alcançavam outra inflorescência do mesmo indivíduo. Com menor freqüência, a visita ocorria em apenas uma das inflorescências, comportamento típico de Bombus morio. Durante as visitas as abelhas tocavam as anteras e os estigmas com a cabeça e parte ventral do corpo. 
Tabela 3. Visitantes florais de Rourea induta em uma área de cerrado sentido restrito na Reserva Ecológica do IBGE, Distrito Federal, Brasil. (Comp. = comprimento do corpo em milímetros; I = entre 8h00 e 9h00; II = entre $10 \mathrm{~h} 30$ e 11h30; III = entre $14 \mathrm{~h} 30$ e $15 \mathrm{~h} 30 ; \mathrm{IV}=$ entre $17 \mathrm{~h} 00$ e $18 \mathrm{~h} 00 ; \mathrm{NI}=$ Espécie não identificada).

Table 3. Floral visitors of Rourea induta in a cerrado strictu sensu area at Reserva Ecológica do IBGE, Distrito Federal, Brazil. $($ Comp = body length $(\mathrm{mm}) ; \mathrm{I}=$ between 8:00 am and 9:00 am; II = between 10:30 am and 11:30 am; III = between 2:30 $\mathrm{pm}$ and 3:30 pm; IV = between 5:00 pm and 6:00 pm; NI = non-identified species).

\begin{tabular}{|c|c|c|c|c|c|c|c|c|c|c|}
\hline \multirow[t]{2}{*}{ Grupo/Família/Espécie } & \multirow[t]{2}{*}{ Comp. } & \multicolumn{4}{|c|}{$\begin{array}{l}\text { Planta } 01 \\
\text { Longistilo }\end{array}$} & \multicolumn{4}{|c|}{$\begin{array}{l}\text { Planta } 02 \\
\text { Brevistilo }\end{array}$} & Tota \\
\hline & & I & II & III & IV & I & II & III & IV & \\
\hline \multicolumn{11}{|l|}{ Abelhas } \\
\hline \multicolumn{11}{|l|}{ APIDAE } \\
\hline Apis mellifera Linnaeus & 11 & 1 & 3 & & & 4 & 2 & & & 10 \\
\hline Bombus morio Swederus & 15 & 1 & & & & & & & & 1 \\
\hline Geotrigona sp. & 6 & & 1 & & & & & & & 1 \\
\hline Paratrigona lineata (Lepeletier) & 5 & & 13 & 4 & & & 2 & 2 & & 21 \\
\hline Scaptotrigona postica Latreille & 6 & 3 & 17 & 6 & & 5 & 8 & 5 & & 44 \\
\hline Tetragona cf. clavipes (Fabricius) & 6 & & 4 & & & & 1 & & & \\
\hline Trigona spinipes (Fabricius) & 7 & & 2 & & 1 & 1 & & & & 4 \\
\hline Trigona sp. & 7 & 1 & & & & & & & & \\
\hline Trigonisca $\mathrm{sp}$. & 2,5 & & 1 & & & & & & & \\
\hline \multicolumn{11}{|l|}{ HALICTIDAE } \\
\hline NI & 5 & & 1 & & & & & & & \\
\hline
\end{tabular}

Moscas

MUSCIDAE

NI

SYRPHIDAE

NI 1

NI 2

TACHINIDAE

NI

Borboletas

HESPERIDAE

Polites vibex catilina Plötz $\quad 14$

Urbanus sp. 16

$16-1$

LYCAENIDAE

NI

NYMPHALIDAE

Junonia evarete Stoll

Vespas

VESPIDAE

Brachygastra sp. $\quad 8$

NI 1

$13-1$

$1 \quad 1 \quad 2$

NI 2

16

Total (indivíduos)

Total (espécies)

$\begin{array}{llll}7 & 45 & 10 & 1\end{array}$

$\begin{array}{llll}13 & 17 & 10 & 0\end{array}$

103

$\begin{array}{lllllllll}5 & 10 & 2 & 1 & 6 & 8 & 5 & 0 & 21\end{array}$



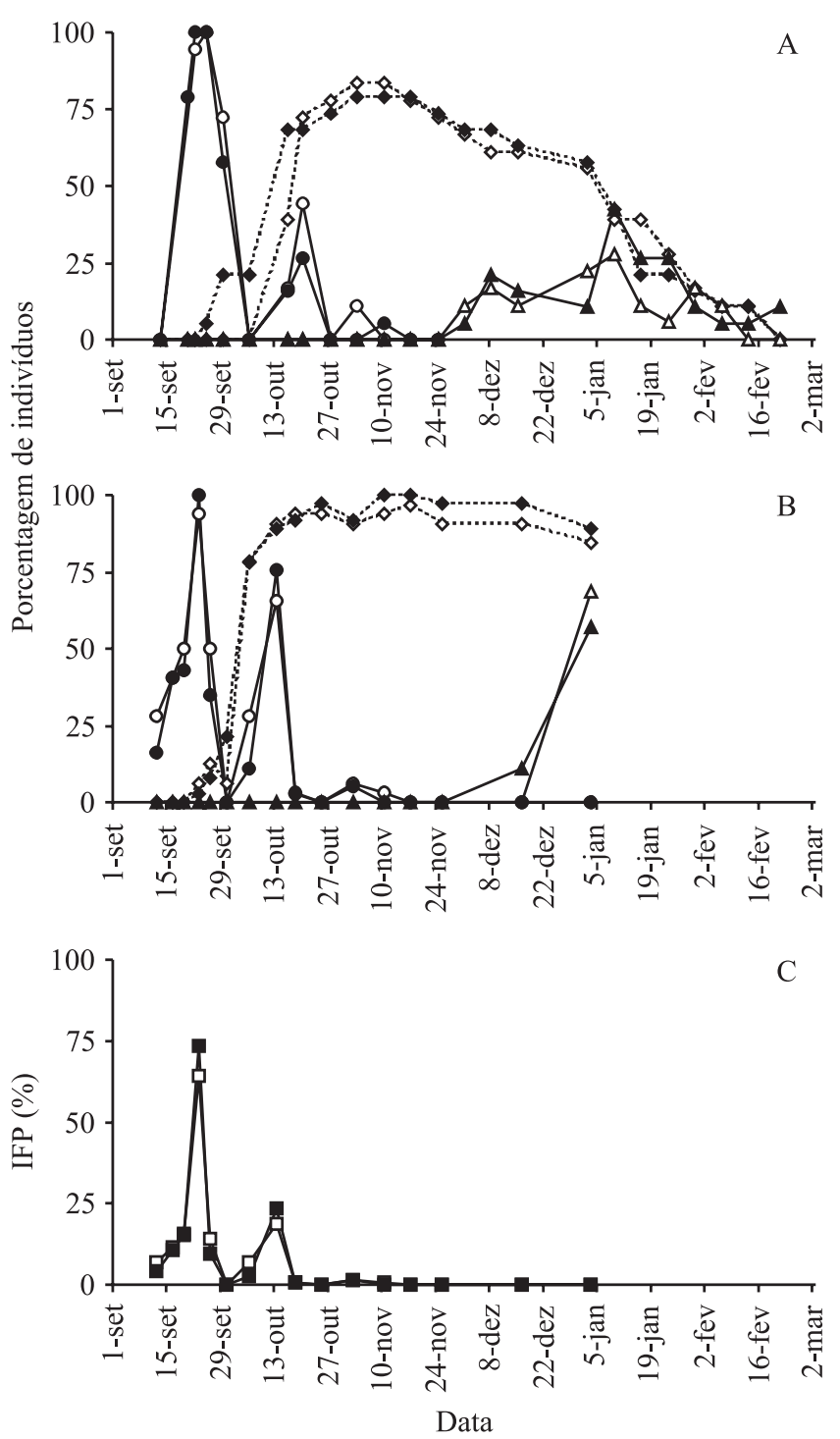

Figura 2. Fenologia reprodutiva de uma população de Rourea induta em uma área de cerrado sentido restrito na Reserva Ecológica do IBGE, Distrito Federal, Brasil $(\bullet=$ flor; $\bullet=$ fruto imaturo; $\boldsymbol{\Delta}$ = fruto maduro; $\boldsymbol{\nabla}=$ intensidade de floração da população (IFP)). A = anos 2000-2001; B e C = anos 20052006. Símbolos abertos representam o morfo brevistilo; símbolos fechados representam o morfo longistilo.

Figure 2. Reproductive phenology of a Rourea induta population in a cerrado strictu sensu area at Reserva Ecológica do IBGE, Distrito Federal, Brazil ( $\bullet=$ flower; $\diamond=$ imature fruit; $\Delta=$ ripe fruit; $\boldsymbol{\square}=$ population flowering intensity (IFP)). $\mathrm{A}=$ years 2000-2001; B and C = years 2005-2006. Open symbols represent thrum morph; full symbols represent pin morph.

\section{Discussão}

Flores actinomorfas, com morfologia simples, pequenas, planas, dialipétalas, dialicarpelares, com verticilos reprodutivos exsertos e estames heterodínamos observadas em $R$. induta, são características compartilhadas por outras espécies heterostílicas da família Connaraceae (Forero et al. 1984, Lemmens 1989, Lloyd \& Webb 1992, Milhomens \& Proença 2002). No entanto, a morfologia floral de $R$. induta difere daquela mais complexa apresentada por espécies heterostílicas, como corola gamopétala e tubular, com verticilos reprodutivos geralmente pouco expostos aos agentes polinizadores (Ganders 1979a).

Embora a heterostilia seja geralmente caracterizada pela hercogamia recíproca e associada a um sistema de incompatibilidade intramorfo (Baker 1962, Lemmens 1989, Barrett 1992), podem ocorrer variações morfológicas individuais e populacionais quanto ao posicionamento recíproco dos verticilos reprodutivos (Richards \& Koptur 1993, Washitani et al. 1994, Consolaro et al. 2005) e, ainda, a perda parcial ou completa nos sistemas de incompatibilidade intramorfo em um ou ambos os morfos (Ornduff 1964, Ganders 1979a, Charlesworth \& Charlesworth 1979, Washitani et al. 1994, Ree 1997). Estas variações morfológicas e genéticas podem garantir certos níveis de polinização e fertilização intramorfo, conforme observado em algumas espécies distílicas (Sobrevila et al. 1983, Barrett \& Cruzan 1994, Barros 1998), bem como em $R$. induta.

Considerando que Connaraceae é a família com maior variação na expressão da heterostilia e que a condição distílica já é uma alteração do padrão tristílico original da família (Lemmens 1989), a condição de autocompatibilidade parcial do morfo longistilo em $R$. induta, dada pelo IAI, pode ser entendida como uma possível fragilidade do sistema heterostílico na família. Este fenômeno foi também observado em Byrsocarpus coccineus Schumach \& Thorr por Baker (1962), sugerindo que o relaxamento do sistema de incompatibilidade parece ser comum na família.

Apesar do relaxamento do sistema de incompatibilidade do morfo longistilo e da ausência de hercogamia recíproca perfeita, foi detectada incompatibilidade intramorfo e a isopletia entre os morfos. Estas características são compatíveis com a classificação de $R$. induta como uma espécie distílica. Estudos anteriores também mostram alguns exemplos nos quais ocorre a quebra parcial no sistema de incompatibilidade em um ou ambos morfos (Ornduff 1964, Darwin 1877, Ganders 1979b, Shore \& Barrett 1985, Barrett \& Cruzan 1994, Barros 1998) e a falta de hercogamia recíproca (Richards \& Koptur 1993, Consolaro et al. 2005) sem a descaracterização da condição heterostílica das espécies. 
A produção de frutos em condições naturais (brevistilo $=40,6 \%$ e longistilo $=25,2 \%$ ) sugere um elevado potencial reprodutivo da população estudada e indica que o serviço de polinização é eficaz. Estudos com espécies entomófilas de outras famílias heterostílicas, com flores pequenas e verticilos expostos também mostraram elevado sucesso reprodutivo, como em Erythroxylum havanense Jacq. (brevistilo $=14 \%$ a $20,1 \%$, longistilo $=5,4 \%$ a $14,3 \%$ ) (Domínguez et al. 1997); Erythroxylum campestre A. St.-Hil. (brevistilo $=70 \%$ e longistilo $=78 \%$ ), Erythroxylum suberosum A. St.-Hil. (brevistilo $=26 \%$ e longistilo $=$ $30 \%$ ) e Erythroxylum tortuosum Mart. (brevistilo $=60 \%$ e longistilo $=72 \%)($ Barros 1998); Psychotria barbiflora DC. $($ brevistilo $=28 \%$, longistilo $=14,6 \%)($ Teixeira $\&$ Machado 2004); Cordia multispicata (48\%, indistintamente para os dois morfos) (Machado \& Loiola 2000).

A produção de frutos no tratamento controle e o IER em $R$. induta foram maiores no morfo brevistilo, apesar de certo nível de autocompatibilidade intramorfo observada no morfo longistilo. Segundo Ganders (1974), Ganders (1979a) e Dulberger (1992), a maior exposição dos estames em flores brevistilas e dos estigmas em flores longistilas promove maiores níveis de polinização cruzada no morfo brevistilo. Este mecanismo, pode explicar o maior sucesso reprodutivo e a maior eficiência dos polinizadores observados para o morfo brevistilo em $R$. induta.

Apesar das diferenças morfológicas e do sistema reprodutivo entre os morfos florais, o número de frutos maduros produzidos por plantas nos anos de 2000 e 2005 e a taxa de germinação das sementes foram iguais entre os dois morfos de $R$. induta. Estes resultados indicam que a contribuição sexuada para o recrutamento de novos indivíduos na população estudada é semelhante entre os dois morfos.

A maior freqüência de visitas de abelhas pela manhã pode ter ocorrido em resposta à maior disponibilidade de recursos florais (néctar e pólen) neste período, uma vez que a antese ocorreu até às 10h00. Possivelmente, a antese das flores no intervalo entre $18 \mathrm{~h} 00$ e $8 \mathrm{~h} 00$ ocorreu no início da manhã, aumentando a disponibilidade de recursos florais no período matutino. A ausência de abertura de flores nos demais períodos do dia e a intensa atividade dos visitantes florais pela manhã pode ter reduzido os recursos florais e, conseqüentemente, a atração de visitantes no período vespertino. No campo, foi notada intensa atividade de A. mellifera, durante toda a tarde, visitando as flores de indivíduos de Sclerolobium paniculatum Vogel (Leguminosae) próximos às plantas de $R$. induta amostradas. Assim, pelo menos para A. mellifera, as abelhas poderiam estar visitando preferencialmente as flores de $S$. paniculatum à tarde em busca de uma fonte mais abundante de recursos. Embora não tenham sido feitas observações noturnas de visitantes florais de $R$. induta, acreditamos que estes sejam poucos frequientes ou mesmo ausentes. As flores pequenas e brancas, em forma de pincel, com verticilos reprodutivos expostos e presença de pólen e néctar, caracterizam síndrome de melitofilia (Momose et al. 1998) e confirmam o hábito diurno dos visitantes florais observados em $R$. induta.

A curta longevidade das flores de $R$. induta (inferior a 2 dias) e a alta sincronia de floração em nível individual e populacional aumentam a exposição floral e tornam os recursos (pólen e néctar) abundantes e disponíveis aos polinizadores por um limitado espaço de tempo. Plantas com floração massiva, como $R$. induta, atraem visitantes com comportamento oportunista, capazes de responder rapidamente à disponibilidade efêmera de recursos (Gentry 1974; Bawa 1980; Barrett \& Shore 1985). Este é o caso das pequenas abelhas sociais com capacidade de recrutamento (Kerr 1960, Faegri \& van der Pij1 1976, Newstrom et al. 1994), os principais visitantes florais das flores de $R$. induta. Tais visitantes possuem ainda área de forrageamento e movimento restritos entre plantas, visitando muitas flores e permanecendo por longo tempo em um mesmo indivíduo (Augspurger 1980, Stephenson 1982). Este comportamento aumenta os níveis de autopolinização (Stephenson 1982, Barrett \& Shore 1985, Barrett 1998) e foi observado entre os visitantes florais mais abundantes e potenciais polinizadores de $R$. induta.

A distilia é interpretada como um polimorfismo floral que reduz os níveis de autopolinização e promove a polinização dissortativa (Ganders 1979a, Lloyd \& Webb 1992, Barrett et al. 2000). Em R. induta, a autoincompatibilidade parcial e a incompatibilidade intramorfo foram eficientes para reduzir os níveis de autogamia e determinar a produção de frutos. Adicionalmente, apesar da falta de hercogamia completa, as diferenças nas alturas dos verticilos reprodutivos entre os dois morfos parecem ter contribuído para redução dos níveis de autopolinização intramorfo.

Tomados em conjunto, estes resultados sugerem que $R$. induta é altamente dependente dos agentes polinizadores para realizar o fluxo de pólen entre os morfos e aumentar o sucesso reprodutivo da espécie. Segundo Ganders (1979a), Lloyd \& Webb (1992) e Barrett et al. (2000), o acasalamento entre morfos leva à isopletia em populações de espécies distílicas. Dessa forma, apesar de certo relaxamento no sistema de incompatibilidade em $R$. induta, a produção de sementes na espécie é dependente da polinização cruzada intermorfo que 
garante, em última análise, a razão isoplética encontrada na população estudada.

Agradecimentos - Os autores agradecem à direção da Reserva Ecológica do IBGE (Recor) por autorizarem o trabalho de campo. A José Roberto Rodrigues Pinto, Moacyr B. Dias-Filho e Wagner de Melo Ferreira pelas contribuições de grande valia, durante a elaboração do manuscrito. A Marcelino Chanpagnat Boaventura e aos professores Paulo César Mota, Ivone Rezende Diniz, Kiniti Kitayama pelas identificações dos insetos. A Dra. Antonieta Salomão pelo auxílio para realização dos testes de germinação e por ter disponibilizado o Laboratório de Sementes da Embrapa Recursos Genéticos e Biotecnologia.

\section{Referências bibliográficas}

AUGSPURGER, C.K. 1980. Mass-flowering of a tropical shrub (Hybanthus prunifolius): influence on pollinator attraction and movement. Evolution 34:475-488.

BAKER, H.G. 1962. Heterostyly in the Connaraceae with special reference to Byrsocarpus coccineus. Botanical Gazette 123:206-211.

BAKER, H.G. 1966. The evolution, functioning and breakdown of heteromorphic incompatibility systems I. The Plumbaginaceae. Evolution 18:507-512.

BARRETT, S.C.H. 1992. Heterostylous genetic polymorphisms: model systems for evolutionary analysis. In Evolution and function of heterostyly (S.C.H. Barrett, ed.). SpringerVerlag, Berlin, p.1-24.

BARRETT, S.C.H. 1998. The evolution of mating strategies in flowering plants. Trends in Plant Science 3:335-341

BARRETT, S.C.H. 2002. The evolution of plant sexual diversity. Nature Reviews Genetics 3:237-284.

BARRETT, S.C.H. \& CRUZAN, M.B. 1994. Incompatibility in heterostylous plants. In Genetic control of selfincompatibility and reproductive development in flowering plants (E.G. Williams, A.E. Clarke \& R.B. Knox, eds.). Kluwer Academic Publishers, Dordrecht, p.189-219.

BARRET, S.C.H. \& SHORE, J.S. 1985. Dimorphic incompatibility in Turnera hermannioides Camb. (Turneraceae). Annals of the Missouri Botanical Garden 72:259-263.

BARRETT, S.C.H., JESSON, L.K. \& BAKER, A.M. 2000. The Evolution and function of stylar polymorphisms in flowering plants. Annals of Botany 85:253-265.

BARROS, M.G. 1998. Sistemas reprodutivos e polinização em espécies simpátricas de Erythroxylum (Erythroxylaceae) do Brasil. Revista Brasileira de Botânica 21:159-166.

BAWA, K.S. 1980. Evolution of dioecy in flowering plants. Annual Review of Ecology and Systematics 11:15-39.

BAWA, K.S. \& BEACH, J.H. 1983. Self-incompatibility systems in the Rubiaceae of a tropical lowland wet forest. American Journal of Botany 70:1281-1288.
BENCKE, C.S.C. \& MORELLATO, L.P.C. 2002. Comparação de dois métodos de avaliação da fenologia de plantas, sua interpretação e representação. Revista Brasileira de Botânica 25:269-275.

BULLOCK, S.H. 1985. Breeding systems in the flora of a tropical decidous forest. Biotropica 17:287-301.

CHARLESWORTH, D. \& CHARLESWORTH, B. 1979. The maintenance and breakdown of distyly. The American Naturalist 114:499-513.

CONSOLARO, H., SILVA, E.B. \& OLIVEIRA, P.E. 2005. Variação floral e biologia reprodutiva de Manettia cordifolia Mart. (Rubiaceae). Revista Brasileira de Botânica 28:85-94.

DARWIN, C. 1877. The different forms of flowers on plants of the same species. John Murray, London.

DOMÍNGUEZ, C.A., ÁVILA-SAKAR, G., VÁSQUEZSANTANA, F. \& MÁRQUEZ-GUZMÁN J. 1997. Morph-biased sterility in the tropical distylous shrub Erythroxylum havanense (Erythroxylaceae). American Journal of Botany 8:626-632.

DULBERGER, R. 1992. Floral polymorphisms and their functional significance in heterostylous syndromes. In Evolution and function of heterostyly (S.C.H. Barrett, ed.). Springer Verlag, Berlin, p.41-84.

FAEGRI, K. \& VAN DER PIJL, L. 1976. The principles of the pollination ecology. Pergamon Press, Oxford.

FORERO, E. 1983. Connaraceae. Flora Neotropica 36:1-207.

FORERO, E., CARBONÓ, E. \& VIDAL, L.A. 1984. Nuevas especies de Connaraceae Neotropicales. Revista Brasileira de Botânica 7:65-77.

FOURNIER, L. 1974. Un método cuantitativo para la medición de características fenológicas en árboles. Turrialba 24:422-423.

GANDERS, F.R. 1974. Disassortative pollination in distylous plant Jepsonia heterandra. Canadian Journal of Botany 52:2401-2406.

GANDERS, F.R. 1979a. The biology of heterostyly. New Zealand Journal of Botany 17:607-635.

GANDERS, F.R. 1979b. Heterostyly in Erythroxylum coca (Erythroxylaceae). Botanical Journal of the Linnean Society 78:11-20.

GANDERS, F.R., DENNY, S.K. \& TSAI, D. 1985. Breeding systems and genetic variation in Amsinckia spectabilis (Boraginaceae). Canadian Journal of Botany 63:533538.

GENTRY, A.H. 1974. Flowering phenology and diversity in tropical Bignoniaceae. Biotropica 6:64-68.

KERR, W.E. 1960. Evolution of communication in bees and its role in speciation. Evolution 14:386-387.

LEMMENS, R.H.M.J. 1989. Heterostyly. In The Connaraceae: a taxonomic study with emphasis on Africa (C.C.H. Jongkind \& R.H.M.J. Lemmens, eds.). Agriculture University Wageningen Papers, Wageningen, p.56-72.

LEWIS, D. 1943. The physiology of incompatibility in plants II. Linum grandiflorum. Annals of Botany 7:115-122. 
LLOYD, D.G. \& WEBB, C.J. 1992. The evolution of heterostyly. In Evolution and function of heterostyly (S.C.H. Barrett, ed.). Springer Verlag, Berlin, p.151178.

MACHADO, I.C. \& LOIOLA, M.I. 2000. Fly pollination and pollinator sharing in two synchronopatric species: Cordia multispicata (Boraginaceae) and Borreria alata (Rubiaceae). Revista Brasileira de Botânica 23:305-311.

MENDONÇA, R.C., FELFILI, J.M., WALTER, B.M.T., SILVA JÚNIOR, M.C., REZENDE, A.V., FILGUEIRAS, T.S. \& NOGUEIRA, P.E. 1998. Flora vascular do Cerrado. In Cerrado: ambiente e flora (S.M. Sano \& S. P. Almeida, eds.). Embrapa Cerrados, Planaltina, p. 289-556.

MILHOMENS, L.C. \& PROENÇA, C.B. 2002. Connaraceae. In Flora do Distrito Federal (T.B Cavalcanti \& A.E. Ramos, eds.). Embrapa Recursos Genéticos e Biotecnologia, Brasília, p.39-49.

MOMOSE, K., YUMOTO, T., NAGAMITSU, T., KATO, M., NAGAMASU, H., SAKAY, S., HARRISON, R.D., ITIOKA, T., HAMID, A.A. \& INOUE, T. 1998. Pollination biology in a lowland dipterocarp forest in Sarawak, Malaysia. I. Characteristics of the plantpollinator community in a lowland dipterocarp forest. American Journal of Botany 85:1477-1501.

NEWSTROM, L.E., FRANKIE, G.W. \& BAKER, H.G. 1994. A new classification for plant phenology based on flowering patterns in lowland tropical rain forest trees at La Selva, Costa Rica. Biotropica 26:141-159.

NUNES, R.V., SILVA JÚNIOR, M.C., FELFILI, J.M. \& WALTER, B.M.T. 2002. Intervalos de classe para abundância, dominância e frequiência do componente lenhoso do cerrado sentido restrito no Distrito Federal. Revista Árvore 26:173-182.

OLIVEIRA, E.C., PIÑA-RODRIGUES, F.C.M., FIGLIOLIA, M.B. 1989. Proposta para padronização da análise de sementes. Revista Brasileira de Sementes 11:1-25.
OLIVEIRA, P.E. \& GIBBS, P.E. 2000. Reproductive biology of woody plants in a cerrado community of Central Brazil. Flora 195:311-329.

ORNDUFF, R. 1964. The breeding system of Oxalis suksdorfii. American Journal of Botany 51:307-314.

PROENÇA, C.E.B. \& GIBBS, P.E. 1994. Reproductive biology of eight sympatric Myrtaceae from Central Brazil. New Phytologist 126:343-354.

REE, R.H. 1997. Pollen flow, fecundity, and adaptative significance of heterostyly in Palicourea padifolia (Rubiacaea). Biotropica 29:298-308.

RICHARDS, J.H. \& KOPTUR, S. 1993. Floral variation and distyly in Guettarda scabra (Rubiaceae). American Journal of Botany 80:31-40.

RUIZ, T.Z. \& ARROYO, M.T.K. 1978. Plant reproductive ecology of a secondary deciduous tropical forest. Biotropica 10:221-230.

SHORE, J.S. \& BARRETT, S.C.H. 1985. The Genetics of distyly and homostyly in Turnera ulmifolia L. (Turneraceae). Heredity 55:167-174.

SOBREVILA, C., RAMIREZ, N. \& DE ENRECH, N.X. 1983. Reproductive biology of Palicourea fendleri and $P$. petiolaris (Rubiaceae), heterostylous shrubs of a tropical cloud forest in Venezuela. Biotropica 15:161-169.

STEPHENSON, A.G. 1982. When does outcrossing occur in a mass-flowering plant? Evolution 36:762-767.

TEIXEIRA, L.A.G. \& MACHADO, I.C. 2004. Biologia da polinização e sistema reprodutivo de Psychotria barbiflora DC. (Rubiaceae). Acta Botanica Brasilica 18:853-862.

VUILLEUMIER, B.S. 1967. The origin and evolutionary development of heterostyly in the angiosperms. Evolution 21:210-226.

WASHITANI, I., OSAWA, R., NAMAI, H. \& NIWA, M. 1994. Patterns of female fertility in heterostylous Primula sieboldii under severe pollinator limitation. Journal of Ecology 82:571-579. 